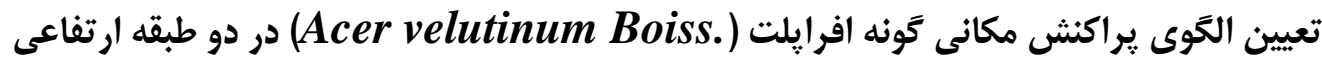

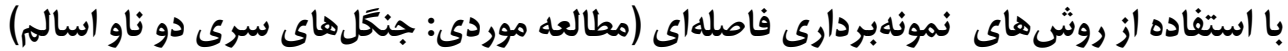

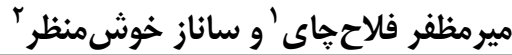

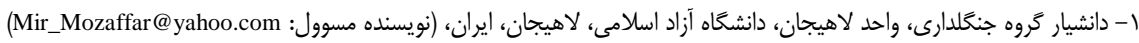

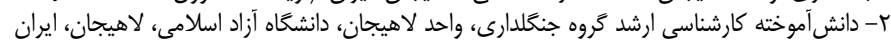

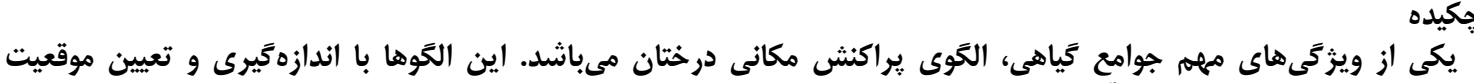

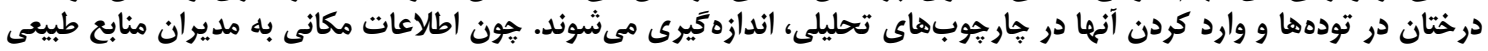

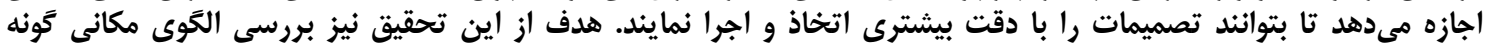

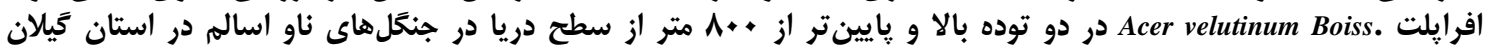

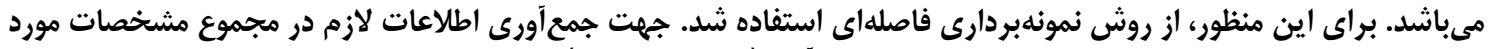

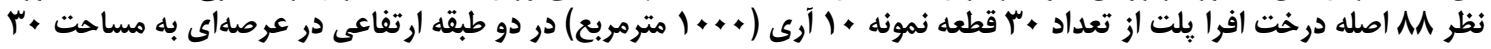

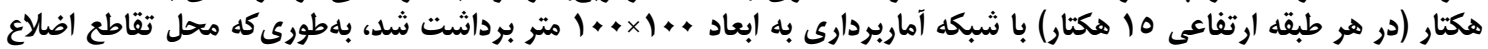

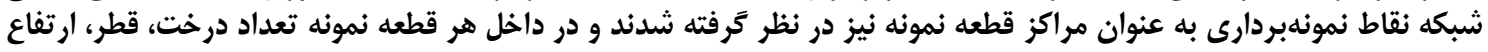

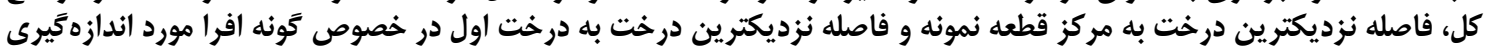

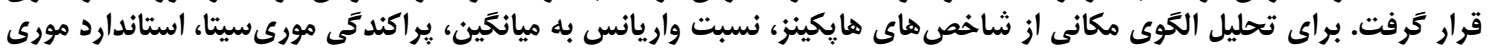

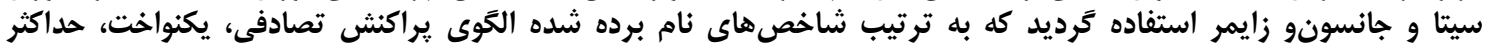

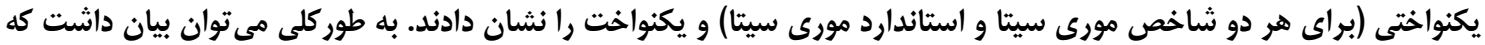

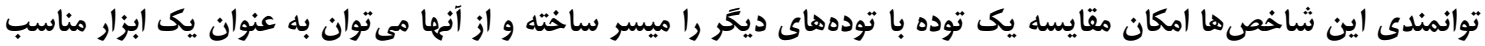

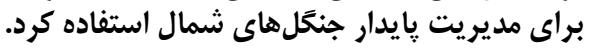

وازههاى كليدى: افرا، الكَىمكانى، نمونه بردارى فاصلهاى، جنگَلهاى اسالم

فارس انجام دادند به اين نتيجه رسيدندكه الخوى مكانى

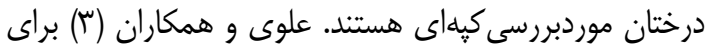

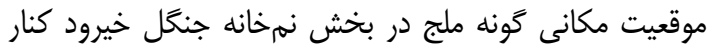

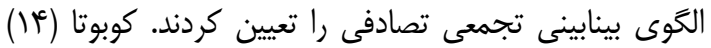

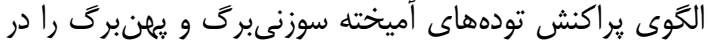

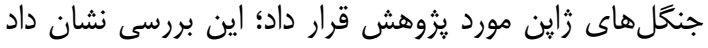

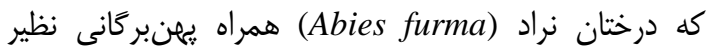
راش (Fagus crenata) افرا (Ace rmicranthum) و و كامليا به صورت كيهاى قرار مى (Camellia japonica)

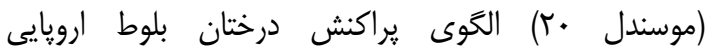

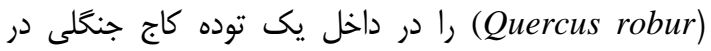

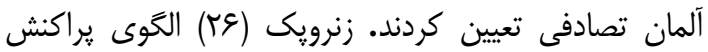

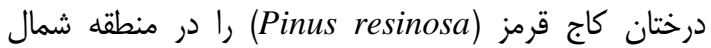

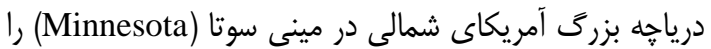

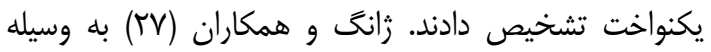

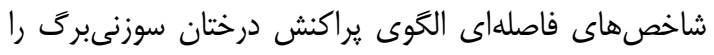

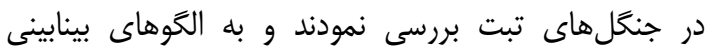

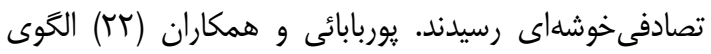

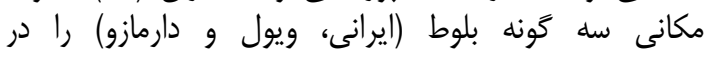

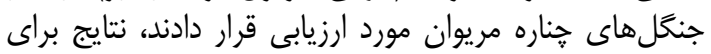

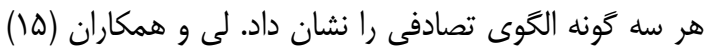

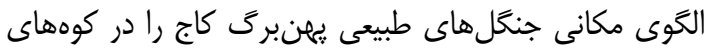

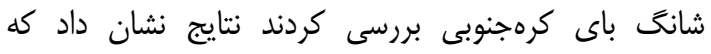

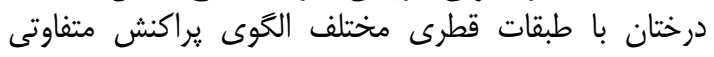

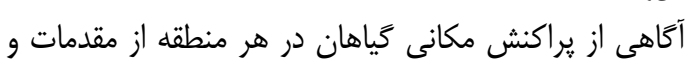

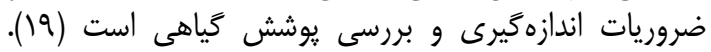

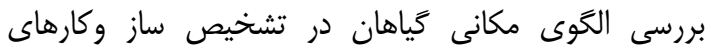

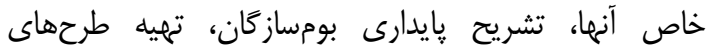

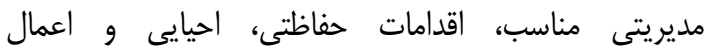

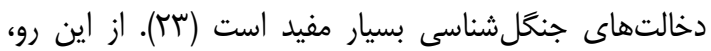

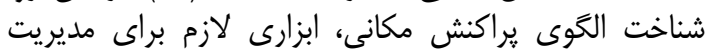

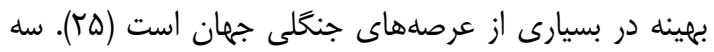

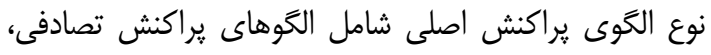

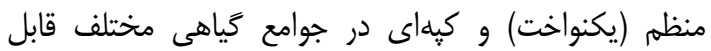

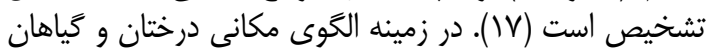

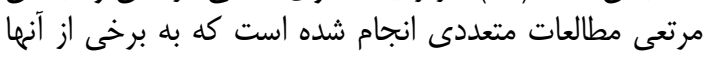

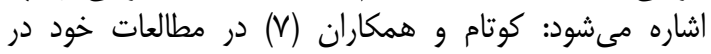

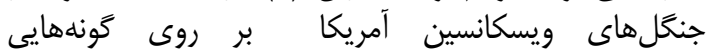
مانند راش آمريكايى (Fagus grandifolia) نراد بالزيكاماني (Tilia americana) و نمدار آمريكايى (Abies balsamea)

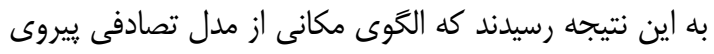

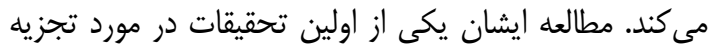

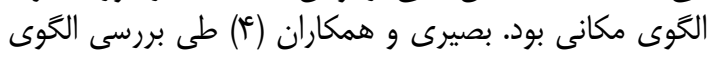

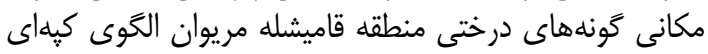

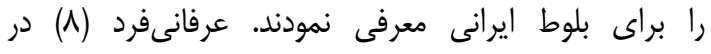

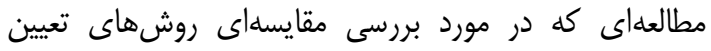

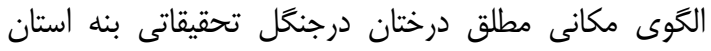


شنى - ليمون رسى شنى و وضعيت بايدارى خاك آن متوسط

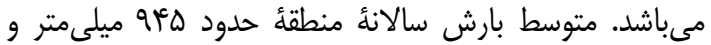

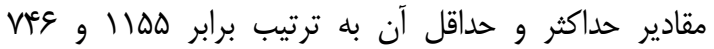

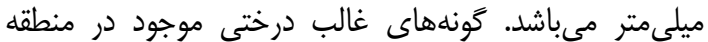

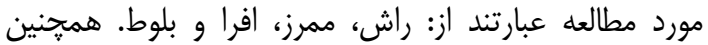

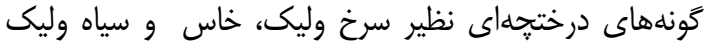

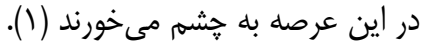
روش تحقيق جهت جمع آورى اطلاعات لازم جمعاً تعداد ·ب قطعه نمونه

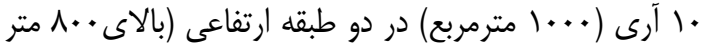

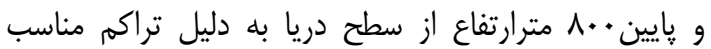

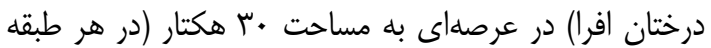

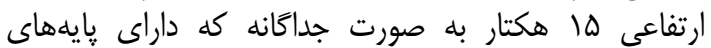

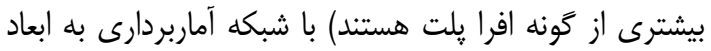

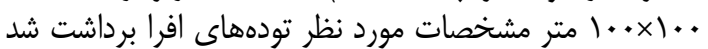

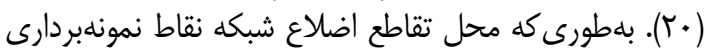

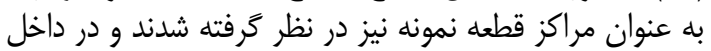

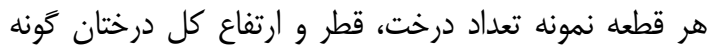

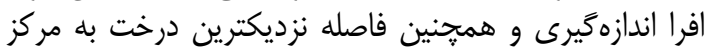

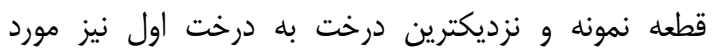

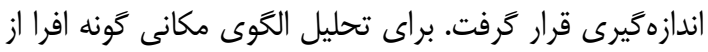

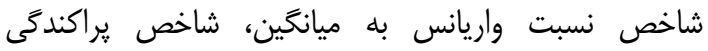

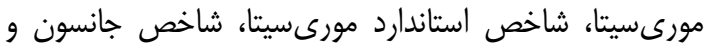

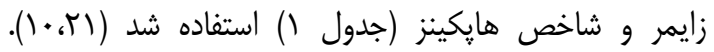
سيس دادهها وارد نرمافزار (Ecological methodology)

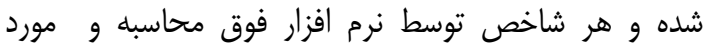
تجزيه و تحليل قرار كرفتند (سا) ترا).
دارند، درختان كوجى داراى الكوى كيهاى و درختان مسن

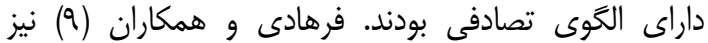

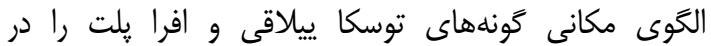

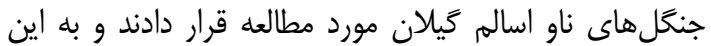

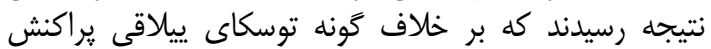

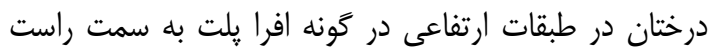

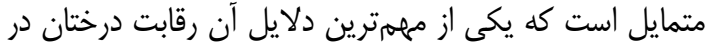

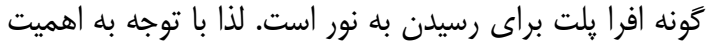

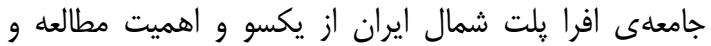

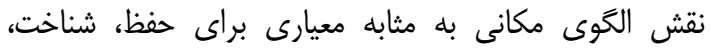

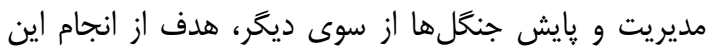

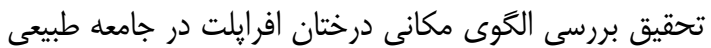

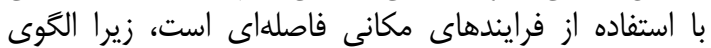

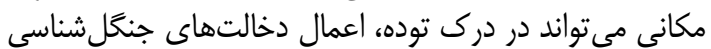
و اقدامات حفاظتى و احيايى بسيار مفيد باشد.

\section{مواد و روشها منطقه مورد مطالعه} اين تحقيق در جنعل هاعل شمال شمال ايران در حوزه آبخيز

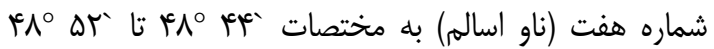

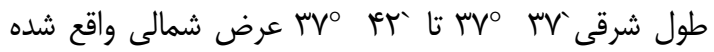

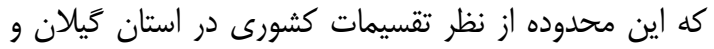

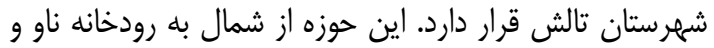

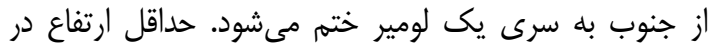

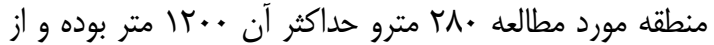

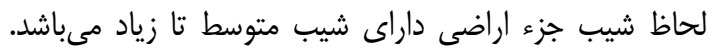
سنگ مادرى سيليسى و نفوذيذيرى سنى ماريب مادرى ليمون

Table 1. Mathematical relationships of the indicators used in this study

جدول 1- روابط رياضى شاخصهاى مورد استفاده دراين مطالعه

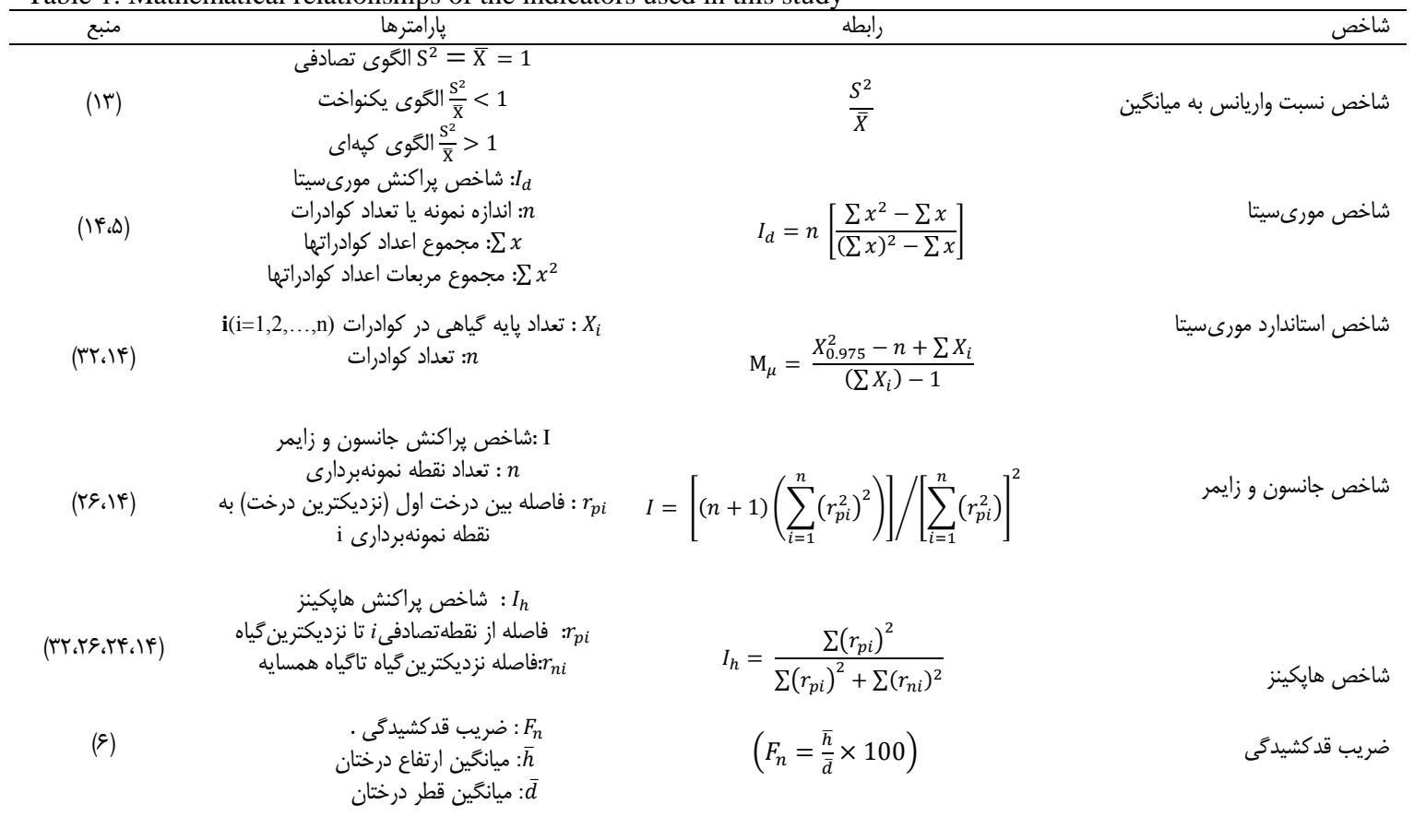




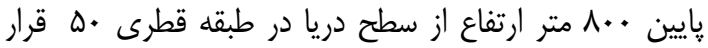

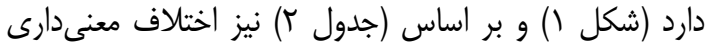
نتايج و بحث

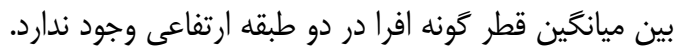

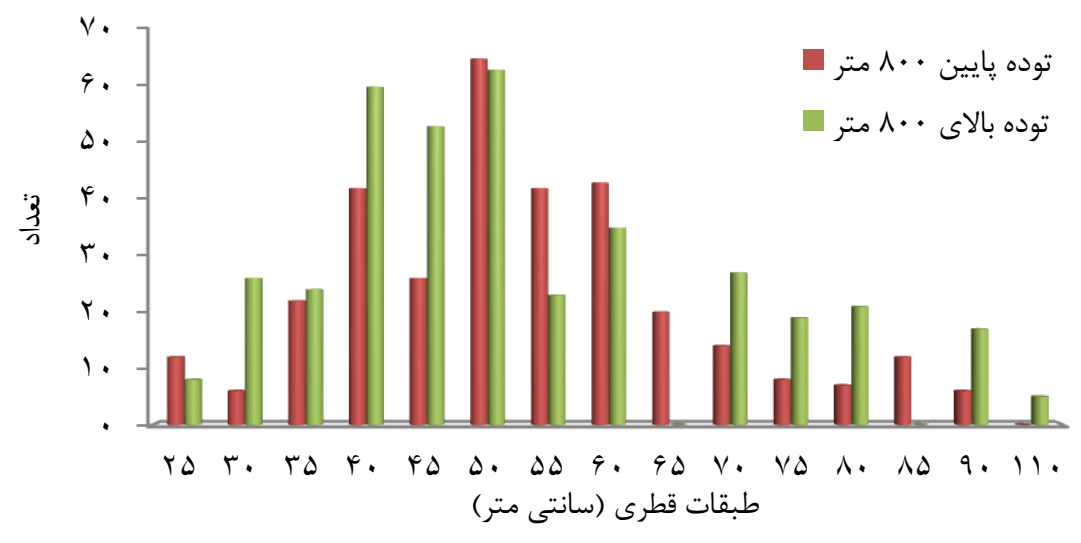

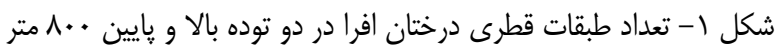

Figure 1. The number of diameter classes of maple trees in two stands $800 \mathrm{~m}$

Table 2. Mean Comparison of diameter of maple species in two altitudes

$$
\text { جدول r- مقايسه ميانخين قطر گَونه افرا در دو طبقه ارتفاعى }
$$

\begin{tabular}{|c|c|c|c|c|c|}
\hline سطح معنى دارى & $\mathrm{t}$ & درجه آزادى & اختاف خطاى استاندارد & اختلاف ميانگين & مشخصه \\
\hline.$/ 9 \gamma^{m^{n s}}$ &.$/ . M F$ & $V \cdot f$ & $1 / T K$ & .1 .41 & قطر \\
\hline
\end{tabular}

ارتفاعى •r متر مشاهده شد. همجنين آزمون مقايسه ميانگين

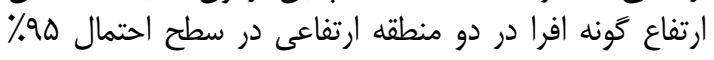
براساس (شكل r) بيشترين تعداد افرا در توده بالاى •.ی

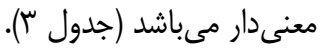

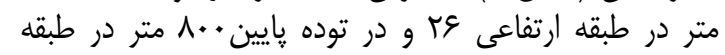

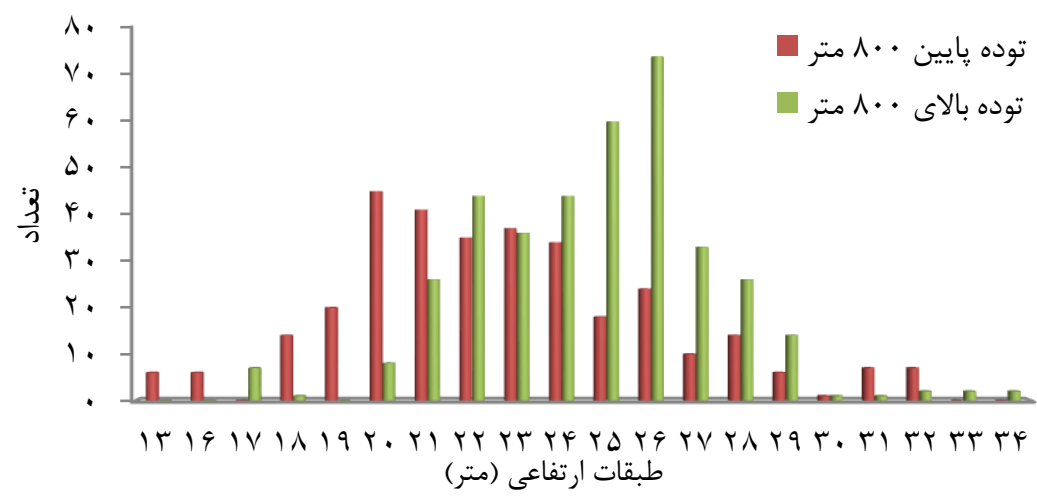

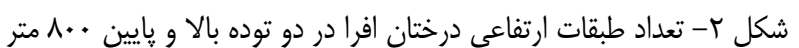

Figure 2. The number of height classes of maple trees in two stands above and below $800 \mathrm{~m}$

Table 3. Mean Comparison of height of maple trees in two altitudes

\begin{tabular}{|c|c|c|c|c|c|}
\hline سطح معنى دارى & $\mathrm{T}$ & درجه آزادى & اختلافخطاى استاندارد & اختلاف ميانكين & مشخصه \\
\hline$\cdot 1 \ldots * *$ & $N / r \cdot r$ & $V \cdot r$ & $\cdot / \pi F$ & 1/9D & 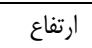 \\
\hline
\end{tabular}




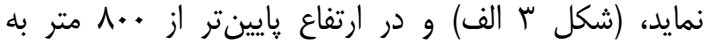

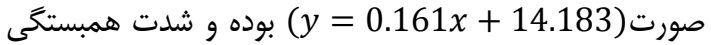
آن $(r=64.22 \%)$ مى مياشد و ضريب تبيين آن نيز

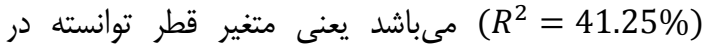

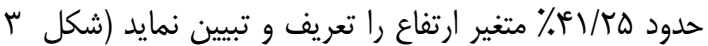

$y=0.161 x+14.183$

$$
R^{2}=0.4125
$$

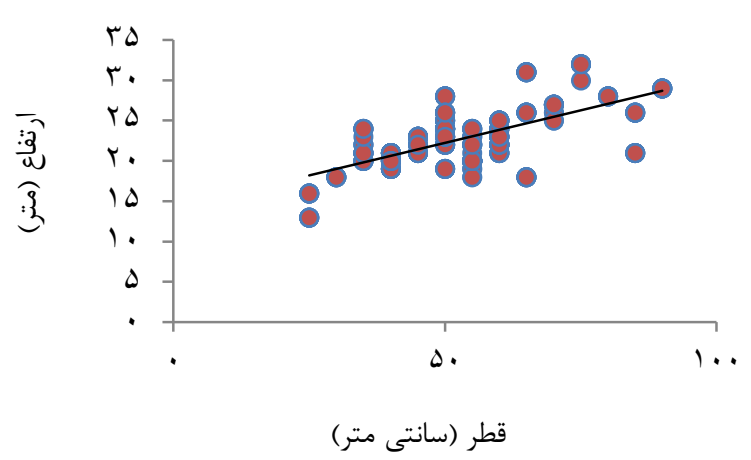

(ب)
ضريب همبستكى بين قطر وارتفاع

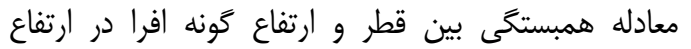

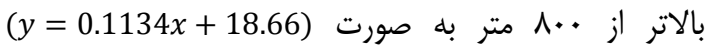

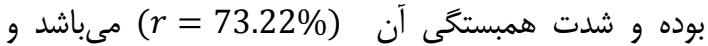
ضريب تبيين آن نيز (

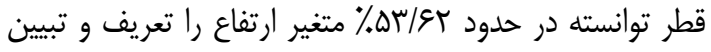

$$
y=0.1134 x+18.669
$$$$
R^{2}=0.5362
$$

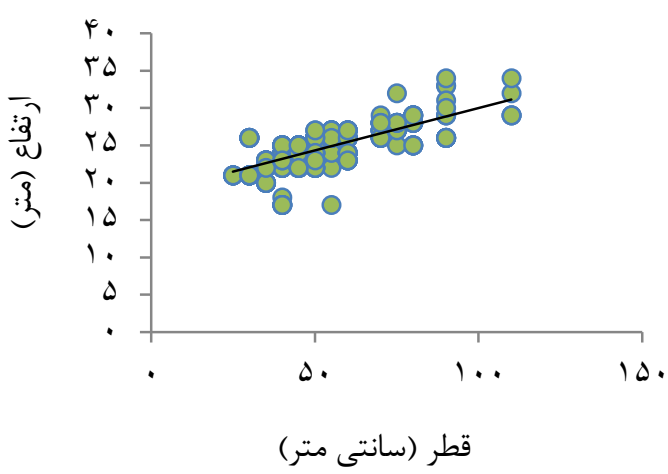

(الف)

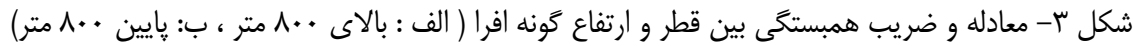

Figure 3. Equation and correlation coefficient between the diameter and height of maple trees (A: above 800 m, B: below $800 \mathrm{~m}$ )

$F_{n}=\frac{\bar{h}}{\bar{d}}=\frac{22.72}{53.05}=0.428 \times 100=42.8 \%$

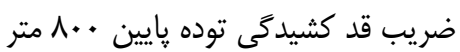

$\mathrm{F}_{n}=\frac{\bar{h}}{\bar{d}}=\frac{24.68}{53}=0.465 \times 100=46.5 \%$ ضريب قد كشيدگى توده بالاى +.
ضريب قد كثيدگى (يايدارى) توده

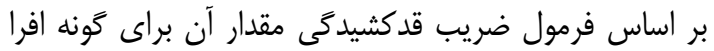

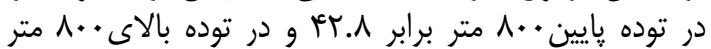

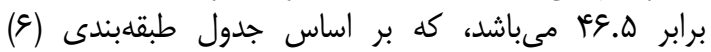

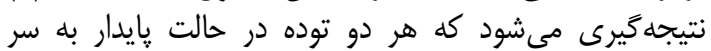

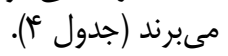

\begin{tabular}{|c|c|c|c|c|}
\hline درخت درفضاى بازروييده & يايدار & ن إيايدار & خيلى نايايدار & ميزان پايدارى \\
\hline$<r$. & $f \cdot-\Lambda$. & $\Lambda \cdot-1 .$. & $>1 \ldots$ & مقدار ضريبd \\
\hline
\end{tabular}
Table 4. Relationship between stability and Burschel and Huss Slimming Factor

جدول ع- رابطه بين پايدارى و ضريب قدكشيدگى بورشل هاس

(y=82.368e $\left.\mathrm{e}^{-0.012 \mathrm{x}}\right)$ (r=82.16\%)

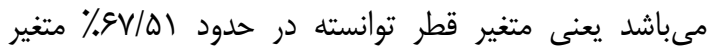

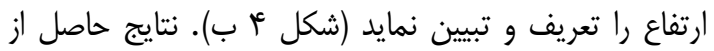

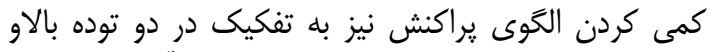

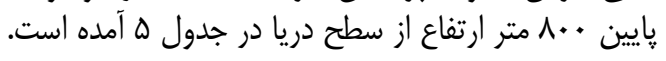

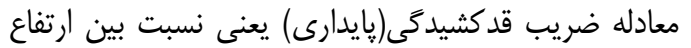

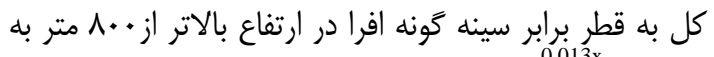
صورت (y=98.885e

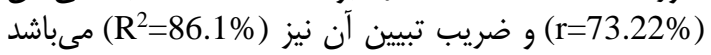

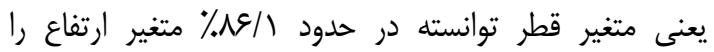

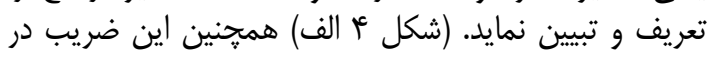

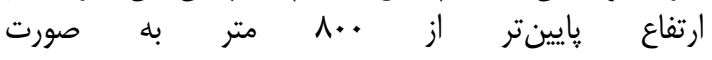




$$
\begin{gathered}
R^{2}=0.6751 \\
y=82.368 e^{-0.012 x}
\end{gathered}
$$

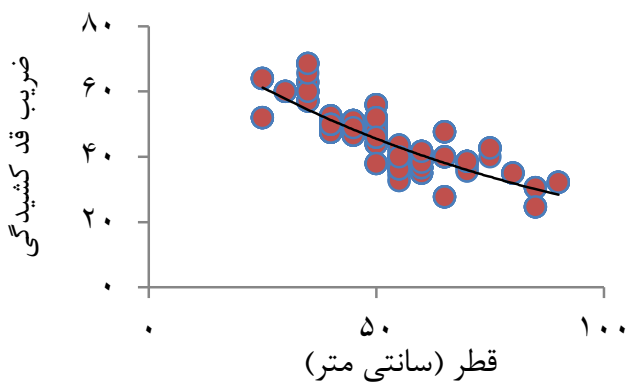

(ب)
$R^{2}=0.861$

$y=98.885 e^{-0.013 x}$

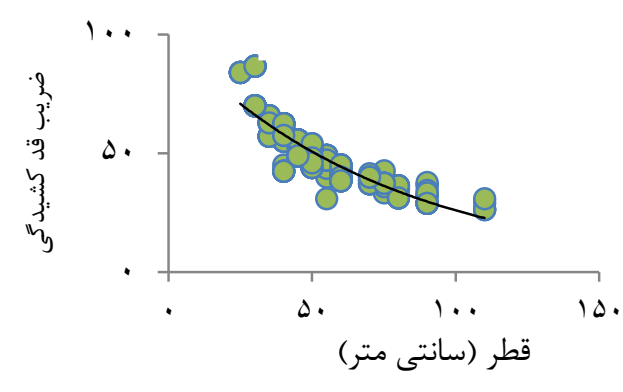

(الف)

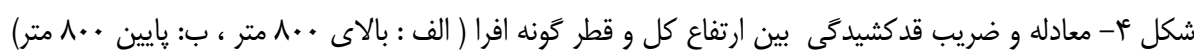

Figure 4. Equation and height coefficient between total height and diameter of maple trees (A: above $800 \mathrm{~m}, \mathrm{~B}$ : below $800 \mathrm{~m}$ )

جدول ه- مقادير شاخصهاى تعيين الخوى براكنش گَونه افرا در منطقه ناو اسالم گيلان

\begin{tabular}{|c|c|c|c|c|}
\hline الخَوى يراكنش & توده پايين •+1 متر & الخَوى براكنش & توده بالاى ..1 متر & شاخص \\
\hline يكنواخت & $\cdot / T V \Delta$ & يكنواخت & $\cdot / q \cdot)^{f}$ & واريانس به ميانكين \\
\hline حداكثر يكنواختى &.$/ 98 V$ & حداكثر يكنواختى &.$/ 998$ & مورى سيتا \\
\hline حداكثر يكنواختى & $\cdot / 191$ & حداكثر يكنواختى &.$/ 1 r q$ & استاندارد مورى سيتا \\
\hline يكنواخت & I/NET & يكنواخت & $1 / 9 \Delta V$ & جانسون و زايمر \\
\hline تصادفى & $\cdot / v \cdot 9$ & تصادفى & . /var & هإِكينز \\
\hline
\end{tabular}
Table 5. The values of indicators for determining the distribution pattern of maple species in the Nave-Aslam

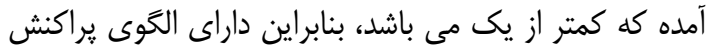

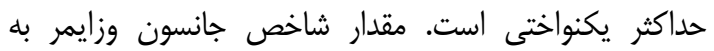

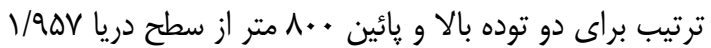

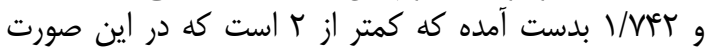

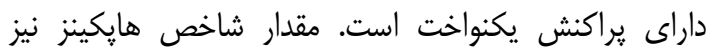

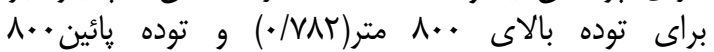

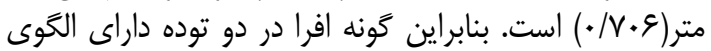

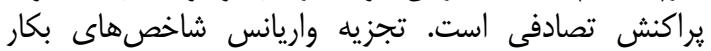

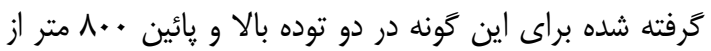

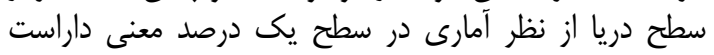

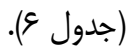

تجزيه واريانس شاخص هاخدا

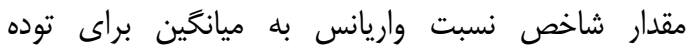

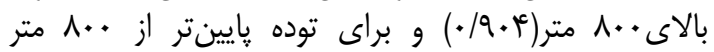

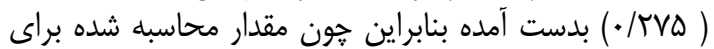

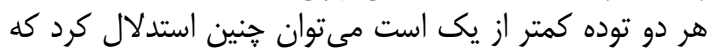

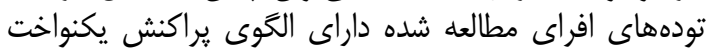

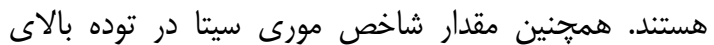

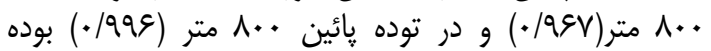

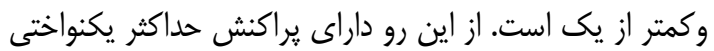

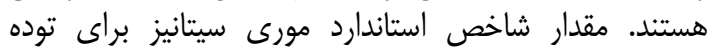

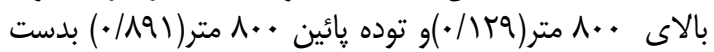

جدول و- تجزيه واريانس شاخصهاى مختلف براى گونه افرا يلت در دو توده

\begin{tabular}{|c|c|c|c|c|}
\hline معنى دارى & مقدار F مق F & ميانگين مربعات & منابع تغييرات & شاخصها \\
\hline \multirow[t]{2}{*}{$\cdot / \ldots^{* w^{*}}$} & $r T / V \cdot \Lambda$ & $1 / \cdot{ }^{*} \Delta$ & تيمار & نسبت واريانس به ميانخين \\
\hline & & .1 .19 & خطا & \\
\hline \multirow[t]{2}{*}{ 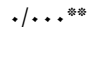 } & $r N / V Y q$ & . /\%q. & 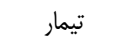 & مورى سيتا \\
\hline & & .1 .14 & خطا & \\
\hline \multirow[t]{2}{*}{$\cdot / \ldots{ }^{* * *}$} & TN/AFT &.$/ \cdot \Delta F$ & تيمار & استاندارد مورى سيتا \\
\hline & &.$/ . r$ & خطا & \\
\hline \multirow[t]{2}{*}{$\cdot 1 \ldots$ *** } &.$/ 191$ &.$/ .4$ & تيمار & جانسون و زاير \\
\hline & & .1 .11 & خطا & \\
\hline \multirow[t]{2}{*}{$\cdot / \ldots$ 橉 } & $1 \cdots / v \cdot 1$ & 1/19. & 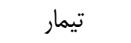 & هايكينز \\
\hline & & $.1 \cdot .1$ & خطا & \\
\hline
\end{tabular}
Table 6. The analysis of variance of different indicators for maple species in two stands 
كه الخوى يراكنش لكههاى زادآورى در سطح توده يكنواخت

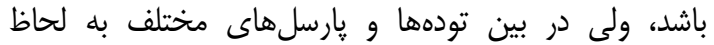

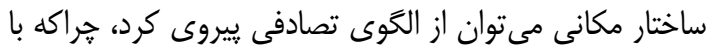

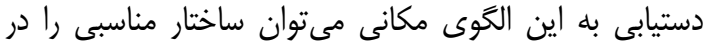

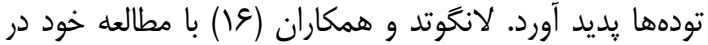

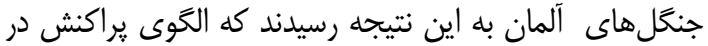

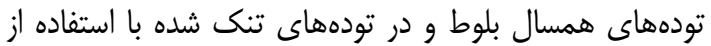

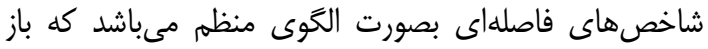

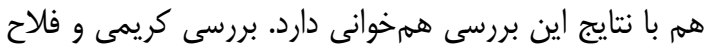

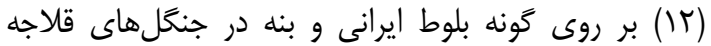

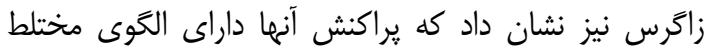

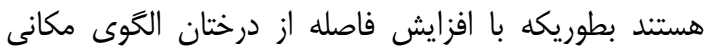

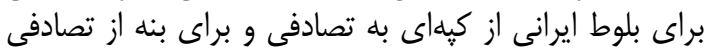

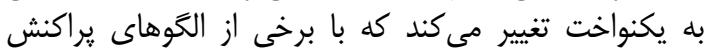

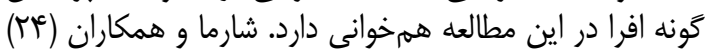

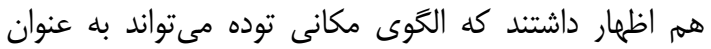

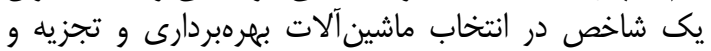

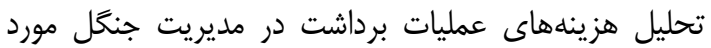

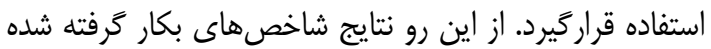

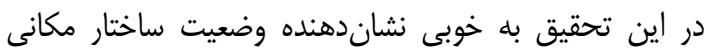

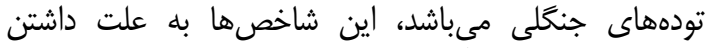

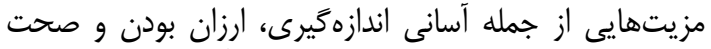

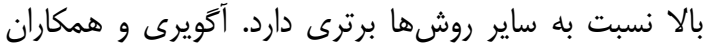

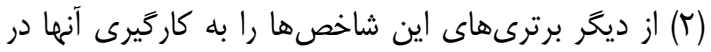

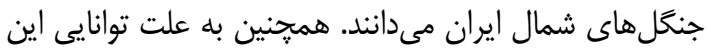

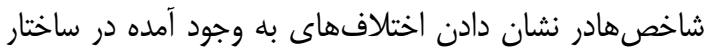

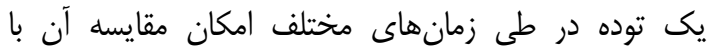

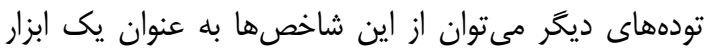

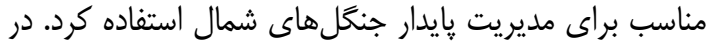

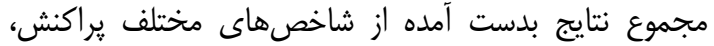

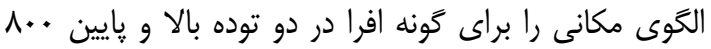

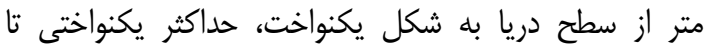
تصادفى انشان مىدهد.

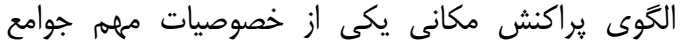

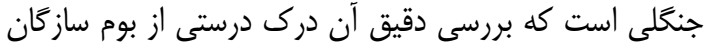

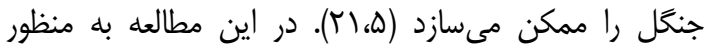

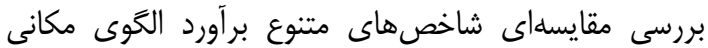

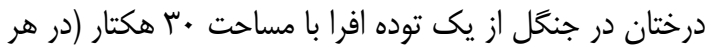

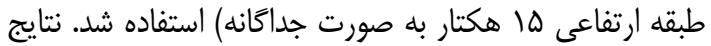

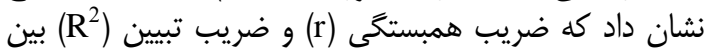

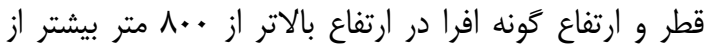

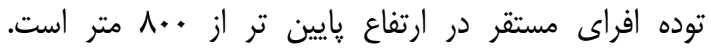

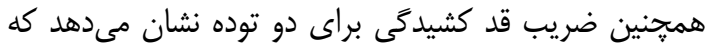

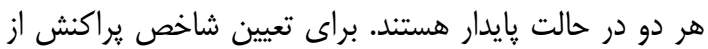

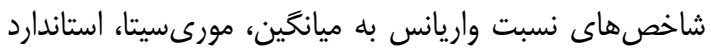
مورىسيتا، جانسون، زايمر و هايكينز استفاده شد، كه به به ترتيب إنيب

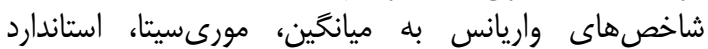

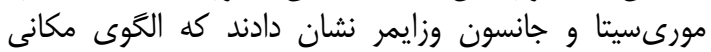

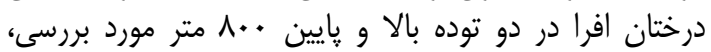

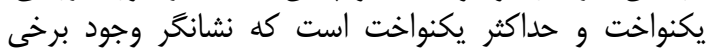

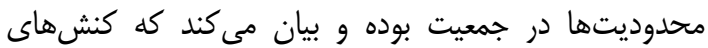

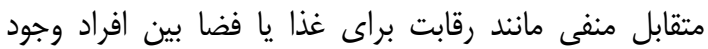

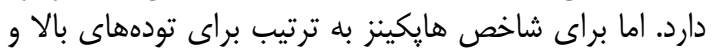

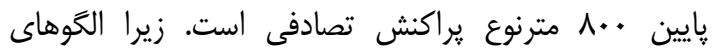

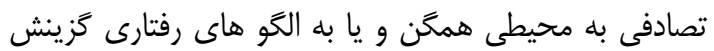

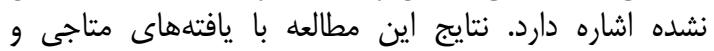

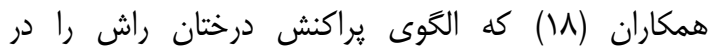

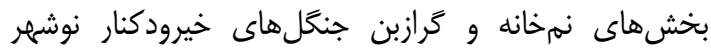

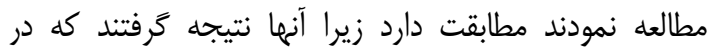

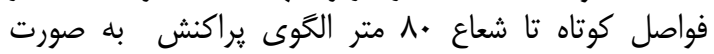

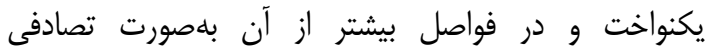

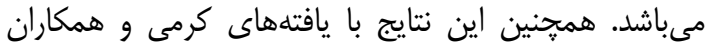

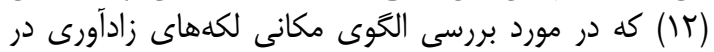

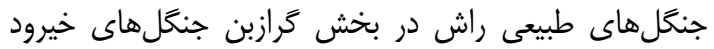

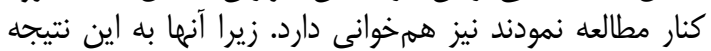

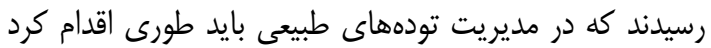

منابع

1. Anonymous. 2003. Nave Asalem forest management project, series 2. Guilan province, Rasht, 125 pp (In Persian).

2. Aguorre, O., G. Hui, K.V. Gadow and J. Jimenes. 2003. An analysis of forest structure using neighborhood-based variables. Forest Ecology and Management, 183: 137-145.

3. Alavi, S.J., G.H. Zahedi Amiri and M.R. Marvi Mohadjer. 2006. An investigation of spatial pattern in WychElm (Ulmus glabra) in Hyrcanian forest, Case study: Kheyroudkenar Forest, Noshahr. Iranian Journal of Natural Resources, 58: 793-804 (In Persian).

4. Basiri, R., H. Sohrabi and M. Mozayen. 2006. A statistical analysis of the spatial pattern of trees species in Ghamisheleh Marivan region. Iran. Journal of the Iranian Natural Resources, 59(2): 579588 (In Persian).

5. Bonham, C.D. 1989. Measurements for terrestrialvegetation. John wiley and sons, 338 pp.

6. Burschel, P. and J. Huss. 1987. Grandsirss des Waldbaus. Verlay paul paarey, Humburg and Berlin.

7. Cottam, G., J.T. Curtis and B.W. Hale. 1953. Some sampling characteristics of a population of randomly dispersed individuals. Ecology, 34: 741-757.

8. Erfanifard, Y. and F. Mahdian. 2012. Comparative investigation on the methods of true spatial pattern analysis of trees in forests, Case study: Wild pistachio research forest, Fars province, Iran. Iranian Journal of Forest andPoplar Research, 20(1): 62-73 (In Persian). 
9. Farhadi, P., J. Sousani and S.Y. Erfanifard. 2015. Efficiency of Uniform Angle Indicators and Distance to Neighbors in Detecting the Spawning Pattern of Alders and Maple Pellets in Aslam Nava Forests in Guilan, Second International Conference on Land Ecology, 9 pp., Isfahan, Isfahan University of Technology.

10. Heidari, R. H. 2008. Distance Sampling Methods inforest Inventory. Razi University Press. 119 pp.

11. Karami, A., J. Feghhi, M. R. Marvie Mohajer and M. Namiranian. 2012. Investigation on thespatial pattern of regeneration patches in natural beech (Fagus orientalis Lipsky) forests (Casestudy: Gorazbon district, Kheiroud- forest). Iranian Journal of Forest, 1(4): 77-87 (In Persian).

12. Karimi, M and A. Fallah. 2017. Spatial Pattern and Interaction Analysis of Quercus Brantii Lindl. And Pistacia Atlantica Desf. In Qalajeh Forests of Kermanshah using K2 Function Ecology of Iranian Forests, 5(9): 9-16 (In Persian).

13. Krebs, C.J. 1999. Ecological methodology. University of Columbia. 620 pp.

14. Kubota, Y. 2007. Spatial pattern an regeneration dynamics in a temperate Abies-Tsuga forest in southwestern Japan. Journalof Forest Research, 11(3): 191-201.

15. Lei, W., Z. Chunyu and Z. Xiuhai. 2009. Spatialpattern of Korean pine broadleaved forest in Changbai Mountains. Scientia Silvae Sinicae, 5: 54-59.

16. Longuetaud, F., S. Thomas, J.M. Leban and H. Pretzch. 2008. Analysis of long-term dynamics of crowns of sessile oake at the stand level by means of spatial statistics. Journal of Forest Ecology and Management, 255: 2007-2019.

17. Ludwig, J.A. and F.J. Reynolds. 1988. Statistical Ecology: A primer on methods and Computing, John Wiley and Sons Press, NewYork, $337 \mathrm{pp}$

18. Mataji, A., S. Babaie-Kafaki, H. Safaee and H. Kiadaliri. 2008. Spatial pattern of regeneration gaps in managed and unmanaged stands in natural Beech (Fagus orientalism) forests. Iranian Journal of Forest and Poplar Research, 16(1): 149-157 (In Persian).

19. Moghaddam, M.R. 2001. Statistical and descriptive ecology of vegetation. Tehran University Press, $285 \mathrm{pp}$.

20. Mosandl, R. and A. Kleinert. 1998. Development of oaks emerged from bird dispersed seeds under old-growth pine stands. Forest Ecology and Management, 106(1): 35-44.

21. Pourbabaei, H., H. Ahani and A.M. Bonyad. 2004. Study on spatial pattern of Acer platanoids L. in Shafarud forests, Rezvanshahr, Guilan. Environment Science Journal, 1: 24-30.

22. Pourbabaei, H., Sh. Zandi Navgaran and M.N. Adel. 2012. Spatial pattern of three oak species in Chenareforest of Marivan, Kordestan. Journal of Natural Environment, 65(3): 329-339.

23. Safari, A., N. Shabanian, R.H. Heydari, S.Y. Erfanifard and M. Pourreza. 2010. Spatial pattern of Manna Oak trees (Quercus brantii Lindl.) in Bayangan forests Kermanshah. Iranian Journal of Forest and Poplar Research, 18(4): 596-608.

24. Sharma, B.D., J. Wang and G. Miller. 2008. A GIS-based approach to stand visualization and spatial pattern analysis in a mixed hardwood forest in West Virginia. In: Jacobs, D.F., Michler, C.H. (Eds.). Proceedings, $16^{\text {th }}$ Central Hardwood Forest Conference, West Lafayette, IN. Gen. Tech. Rep. NRS-P24. U.S. Department of Agriculture, Forest Service, Northern Research Station, Newtown Square, PA, 356- 365 pp.

25. Wulder, M.A. and S.E. Franklin. 2006. UnderstandingForest Disturbance and Spatial Pattern. Remote Sensing and GIS Approach, Taylor \& Francis Group, CRC Press, 243 pp.

26. Zenner, E.K. and J.E. Peck. 2009. Characterizing structural conditions in mature managed red pine: spatial dependency of metrics and adequacy of plot size. Forest ecology and management, 257: 311320.

27.Zhang, Q., Y. Zhang, S. Peng, E. Yirdaw and N. Wu. 2009. Spatial structure of Alpine trees in mountain Baima Xueshan on the southeast Tibetan plateau. Silva Fennica, 43(2): 197-208. 


\title{
Determination of Spatial Distribution Pattern Analysis of Acer Velutinum Species in two Elevation Classes using Distance Sampling Methods (Case Study: Asalem Nav Forests, Series No. 2)
}

\section{Mir Mozaffar FallahChai ${ }^{1}$ and Sanaz Khoshmanzar ${ }^{2}$}

1- Associate Professor, Department of Forestry, Lahijan Branch, Islamic Azad University, Lahijan, Iran, (Corresponding author: Mir_Mozaffar@yahoo.com)

2- Graduated from the Department of Forestry, Lahijan Branch, Islamic Azad University, Lahijan, Iran Received: October 26, $2017 \quad$ Accepted: February 12, 2019

\begin{abstract}
One of the important features of plant communities is the spatial pattern of trees. The spatial pattern of the stands determined by measuring and positioning of trees in the stands and inserting them in analytical frameworks. This is because spatial information allows natural resource managers to make and perform better-informed decisions, -. The aim of this study was to assess the spatial pattern of Acer velutinum in two stands, above and below $800 \mathrm{~m}$ from see level in Asalem Nav forests, Guilan province. The sampling distance- method was used. In order to gather the required data, characteristics of 88 tress in 30 sample plots $\left(1000 \mathrm{~m}^{2}\right)$ were recorded in two elevation classes in an area of 30 ha (15 ha in each elevation class) with a grid network of $100 \times 100$. The number of trees, diameter, height, distance from the nearest tree to the sample plot and distance of the nearest tree to the first tree of maple was measured in each sample plot. To analyze the spatial pattern, the Hopkins index, the variance/average ratio, Morisita dispersion, Morisita, Johnson, and Zimmer standards were used, which randomized, uniform, maximum uniform (for both Morisita and Morisita standard indexes) and uniform patterns dispersion was observed, respectively. In general, the ability of these indices makes it possible to compare the stands and they can be used as a suitable tool for sustainable management of the northern forests of Iran.
\end{abstract}

Keywords: Acer velutinum, Asalem Nav Forests, Spatial Pattern, Sampling Distance Method 